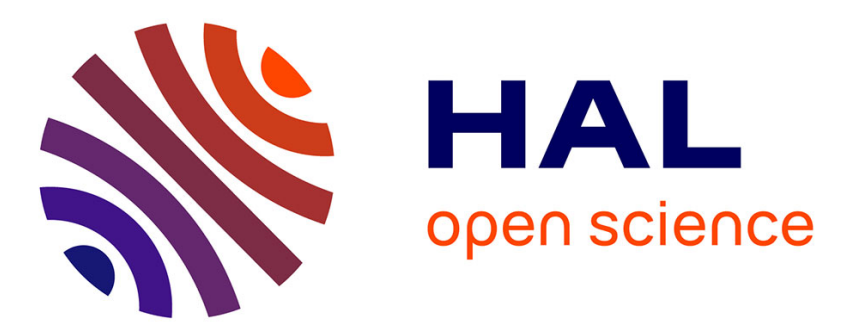

\title{
Available Wrench Set for Planar Mobile Cable-Driven Parallel Robots
}

\author{
Tahir Rasheed, Philip Long, David Marquez-Gamez, Stéphane Caro
}

\section{To cite this version:}

Tahir Rasheed, Philip Long, David Marquez-Gamez, Stéphane Caro. Available Wrench Set for Planar Mobile Cable-Driven Parallel Robots. IEEE International Conference on Robotics and Automation (ICRA 2018), May 2018, Brisbane, Australia. 10.1109/ICRA.2018.8461199 . hal-01757793

\section{HAL Id: hal-01757793 https://hal.science/hal-01757793}

Submitted on 4 Apr 2018

HAL is a multi-disciplinary open access archive for the deposit and dissemination of scientific research documents, whether they are published or not. The documents may come from teaching and research institutions in France or abroad, or from public or private research centers.
L'archive ouverte pluridisciplinaire HAL, est destinée au dépôt et à la diffusion de documents scientifiques de niveau recherche, publiés ou non, émanant des établissements d'enseignement et de recherche français ou étrangers, des laboratoires publics ou privés. 


\title{
Available Wrench Set for Planar Mobile Cable-Driven Parallel Robots
}

\author{
Tahir Rasheed ${ }^{1}$, Philip Long ${ }^{2}$, David Marquez-Gamez ${ }^{3}$ and Stéphane Caro ${ }^{4}$, Member, IEEE
}

\begin{abstract}
Cable-Driven Parallel Robots (CDPRs) have several advantages over conventional parallel manipulators most notably a large workspace. CDPRs whose workspace can be further increased by modification of the geometric architecture are known as Reconfigurable Cable Driven Parallel Robots(RCDPRs). A novel concept of RCDPRs, known as Mobile CDPR (MCDPR) that consists of a CDPR carried by multiple mobile bases, is studied in this paper. The system is capable of autonomously navigating to a desired location then deploying to a standard CDPR. In this paper, we analyze the Static equilibrium (SE) of the mobile bases when the system is fully deployed. In contrast to classical CDPRs we show that the workspace of the MCDPR depends, not only on the tension limits, but on the SE constraints as well. We demonstrate how to construct the Available Wrench Set (AWS) for a planar MCDPR wih a point-mass end-effector using both the convex hull and Hyperplane shifting methods. The obtained results are validated in simulation and on an experimental platform consisting of two mobile bases and a CDPR with four cables.
\end{abstract}

\section{INTRODUCTION}

A Cable-Driven Parallel Robot (CDPR) is a type of parallel manipulator whose rigid links are replaced by cables. The platform motion is generated by an appropriate control of the cable lengths. Such robots hold numerous advantages over conventional robots e.g. high accelerations, large payload to weight ratio and large workspace [2]. However, one of the biggest challenges in classical CDPRs which have a fixed cable layout, i.e. fixed exit points and cable configuration, is the potential collisions between the cables and the surrounding environment that can significantly reduce the workspace. By appropriately modifying the robot architecture, better performance can be achieved [3]. CDPRs whose geometric structure can be changed are known as Reconfigurable Cable-Driven Parallel Robots (RCDPRs). Different strategies, for instance maximizing workspace or increasing platform stiffness, have been proposed to optimize cable layout in recent work on RCDPRs [3]-[6]. However, reconfigurability is typically performed manually, a costly and time consuming task.

Recently a novel concept of Mobile Cable-Driven Parallel Robots (MCDPRs) has been introduced in [1] to achieve

*This work is supported by École Centrale Nantes and Echord++ FASTKIT project.

${ }^{1}$ École Centrale de Nantes, Laboratoire des Sciences du Numérique de Nantes, UMR CNRS 6004, 1, rue de la Noë, 44321 Nantes, France, Tahir.Rasheedels2n.fr

${ }^{2} \mathrm{RIVeR}$ Lab, Department of electrical and computing engineering, Northeastern University, USA p. longenortheastern. edu

${ }^{3}$ IRT Jules Verne, Chemin du Chaffault, 44340, Bouguenais, France, david.marquez-gamezdirt-jules-verne.fr

${ }^{4}$ CNRS, Laboratoire des Sciences du Numérique de Nantes, UMR CNRS 6004, 1, rue de la Noë, 44321 Nantes, France, stephane.carodls2n.fr

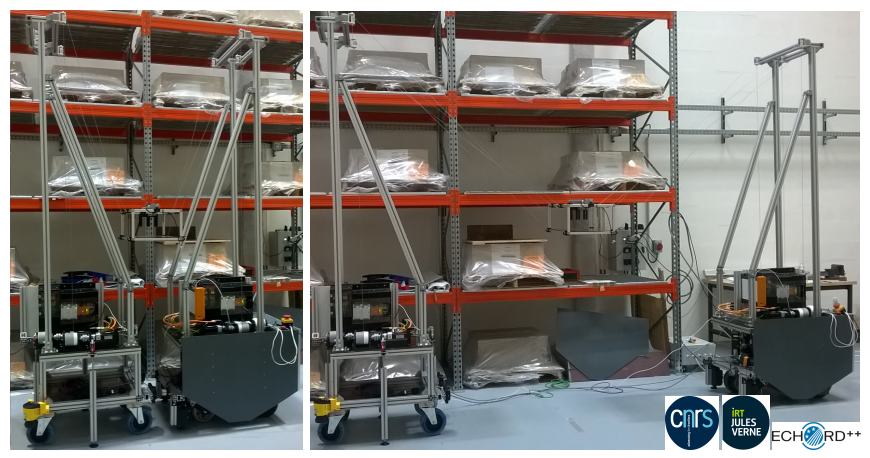

Fig. 1: Fastkit prototype, Undeployed configuration (left) Deployed configuration (right)

an autonomous reconfiguration of RCDPRs. A MCDPR is composed of a classical CDPR with $q$ cables and a $n$ degreeof-freedom (DoF) moving-platform mounted on $p$ mobile bases (MBs). The MCDPR prototype that has been designed and built in the context of Echord++ 'FASTKIT' project is shown in Fig. 1. FASTKIT addresses an industrial need for flexible pick-and-place operations while being easy to install, keeping existing infrastructures and covering large areas. The prototype is composed of eight cables $(q=8)$, a six degree-of-freedom moving-platform $(n=6)$ and two MBs $(p=2)$. The overall objective is to design and implement a system capable of interacting with a high level task planner for logistic operations. Thus the system must be capable of autonomously navigating to the task location, deploying the system such that the task is within the reachable workspace and executing a pick-and-place task. In spite of the numerous advantages of the mobile deployable system, the structural stability must be considered. In [1], a real time continuous tension distribution scheme that takes into account the dynamic equilibrium of the moving-platform and the static equilibrium (SE) of the MBs has been proposed. In this paper, we focus on the workspace analysis of MCDPRs.

The classical techniques used to analyze the workspace of CDPRs are wrench-closure workspace (WCW) [7], [8] and wrench-feasible workspace (WFW) [9]. In this paper, WFW is chosen as it is more relevant from a practical viewpoint. WFW is defined as the set of platform poses for which the required set of wrenches can be balanced with wrenches generated by the cables, while maintaining the cable tension within the defined limits [9]. For a given pose, the set of wrenches a mechanism can generate is defined as available wrench set (AWS), denoted as $\mathscr{A}$. For classical CDPRs, AWS depends on the robot geometric architecture and the tension limits. The set of wrenches required to complete a task, referred to as the required wrench set (RWS), denoted 
as $\mathscr{R}$, will be generated if it is fully included by $\mathscr{A}$ :

$$
\mathscr{R} \subseteq \mathscr{A}
$$

For MCDPRs, the classical definition of AWS for CDPRs must additionally consider the Static Equilibrium (SE) constraints associated with the MBs. The two main approaches used to represent the AWS for CDPRs are the Convex hull method and the Hyperplane shifting method [10]. Once the AWS is defined, WFW can be traced using the Capacity Margin index [11], [12].

This paper deals with the determination of the AWS required to trace the workspace for planar MCDPRs with point-mass end-effector. Figure 2 illustrates a Planar MCDPR with $p=2 \mathrm{MBs}, q=4$ number of cables and $n=2 \mathrm{DoF}$ point mass end-effector. In this paper, wheels are assumed to form a simple contact support with the ground and friction is sufficient to prevent the MBs from sliding. This paper is organized as follows. Section II presents the parameterization of a MCDPR. Section III deals with the SE conditions of the MBs using free body diagrams. Section IV is about the nature of AWS for MCDPRs by considering the SE of the platform as well as the SE of the MBs. Section V discusses how to trace the workspace using the capacity margin index. Section VI shows some experimental validations of the concept. Finally, conclusions are drawn and future work is presented in Section VII.

\section{PARAMETERIZATION OF A MCDPR}

Let us denotes the $j$ th Mobile Base (MB) as $\mathscr{M}_{j}$, $j=1, \ldots, p$. The $i$ th cable mounted onto $\mathscr{M}_{j}$ is named as $\mathscr{C}_{i j}, i=1, \ldots, q_{j}$, where $q_{j}$ denotes the number of cables carried by $\mathscr{M}_{j}$. The total number of cables of the MCDPR is equal to

$$
q=\sum_{j=1}^{p} q_{j}
$$

Let $\mathbf{u}_{i j}$ be the unit vector of $\mathscr{C}_{i j}$ pointing from the pointmass effector to the cable exit point $A_{i j}$. Let $\mathbf{t}_{i j}$ be the cable tension vector along $\mathbf{u}_{i j}$, expressed as

$$
\mathbf{t}_{i j}=t_{i j} \mathbf{u}_{i j}
$$

where $t_{i j}$ is the tension in the $i$ th cable mounted on $\mathscr{M}_{j}$. The force $\mathbf{f}_{i j}$ applied by the $i$ th cable onto $\mathscr{M}_{j}$ is expressed as

$$
\mathbf{f}_{i j}=-t_{i j} \mathbf{u}_{i j}
$$

\section{Static Equilibrium of a Planar MCDPR}

For a planar MCDPR with a point mass end-effector, the SE equation of the latter can be expressed as

$$
\mathbf{f}_{e}=-\sum_{j=1}^{p} \sum_{i=1}^{q_{j}} t_{i j} \mathbf{u}_{i j}
$$

Equation (5) can be expressed in the matrix form as:

$$
\mathbf{W t}+\mathbf{f}_{e}=\mathbf{0},
$$

where $\mathbf{W}$ is a $(2 \times q)$ wrench matrix mapping the cable tension vector $\mathbf{t} \in \mathbb{R}^{q}$ onto the wrench applied by the cables

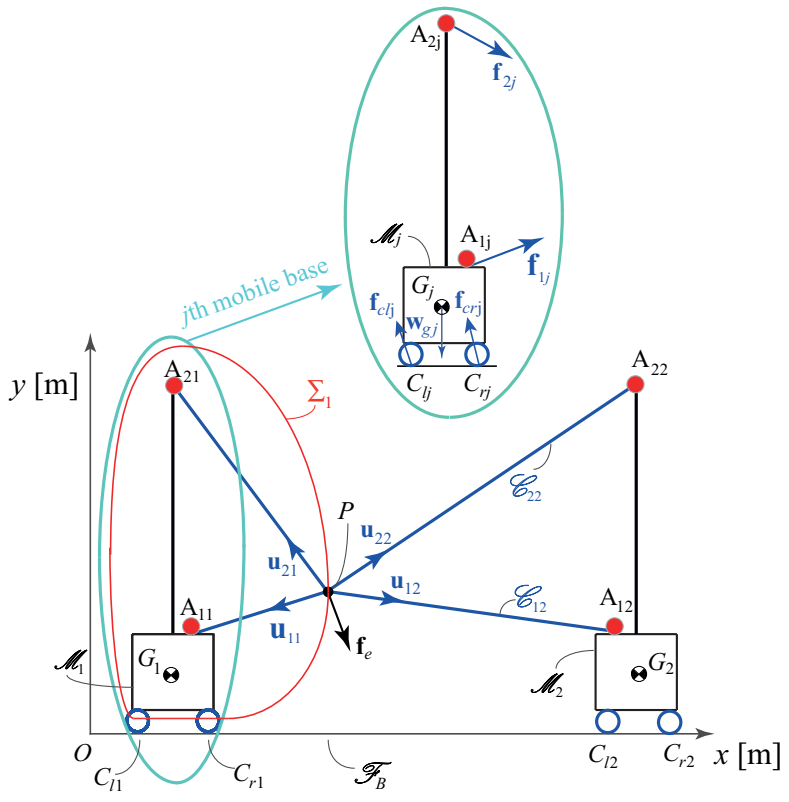

Fig. 2: Planar MCDPR composed of two MBs $(p=2)$, four cables $(q=4)$ with two cables per mobile base $\left(q_{1}=q_{2}=2\right)$ and a two degree-of-freedom $(n=2)$ point-mass end-effector

on the end-effector. $\mathbf{f}_{e}=\left[\begin{array}{ll}f_{e}^{x} & f_{e}^{y}\end{array}\right]^{T}$ denotes the external wrench applied to the end effector. $\mathbf{t}$ and $\mathbf{W}$ can be expressed as:

$$
\begin{gathered}
\mathbf{t}=\left[\begin{array}{llllll}
\mathbf{t}_{1} & \mathbf{t}_{2} & \ldots & \mathbf{t}_{j} & \ldots & \mathbf{t}_{p}
\end{array}\right]^{T} \\
\mathbf{W}=\left[\begin{array}{llllll}
\mathbf{W}_{1} & \mathbf{W}_{2} & \ldots & \mathbf{W}_{j} & \ldots & \mathbf{W}_{p}
\end{array}\right]
\end{gathered}
$$

where

$$
\begin{gathered}
\mathbf{t}_{j}=\left[\begin{array}{llll}
t_{1 j} & t_{2 j} & \ldots & t_{q_{j} j}
\end{array}\right]^{T}, \\
\mathbf{W}_{j}=\left[\begin{array}{llll}
\mathbf{u}_{1 j} & \mathbf{u}_{2 j} & \ldots & \mathbf{u}_{q_{j} j}
\end{array}\right] .
\end{gathered}
$$

As the MBs should be in equilibrium during the motion of the end-effector, we need to formulate the SE conditions for each mobile base, also referred to as the tipping constraints. Figure 2 illustrates the free body diagram of $\mathscr{M}_{j}$ with $q_{j}=2$. The SE equations of $\mathscr{M}_{j}$ carrying up to $q_{j}$ cables is expressed as:

$$
\begin{gathered}
\mathbf{w}_{g j}+\sum_{i=1}^{q_{j}} \mathbf{f}_{i j}+\mathbf{f}_{c l j}+\mathbf{f}_{c r j}=\mathbf{0}, \\
m_{O j}=0 .
\end{gathered}
$$

$\mathbf{w}_{g j}$ denotes the weight vector of $\mathscr{M}_{j} . m_{O j}$ denotes the moment of $\mathscr{M}_{j}$ about point $O . \mathbf{f}_{c l j}=\left[\begin{array}{ll}f_{c l j}^{x} & f_{c l j}^{y}\end{array}\right]^{T}$ and $\mathbf{f}_{c r j}=\left[\begin{array}{ll}f_{c r j}^{x} & f_{c r j}^{y}\end{array}\right]^{T}$ denote the contact forces between the ground and the left and right wheels contact points $C_{l j}$ and $C_{r j}$, respectively. Note that the superscripts $x$ and $y$ in the previous vectors denote their $x$ and $y$ components. $m_{O j}$ can be expressed as:

$$
m_{O j}=\mathbf{g}_{j}^{T} \mathbf{E}^{T} \mathbf{w}_{g j}+\sum_{i=1}^{q_{j}} \mathbf{a}_{i j}^{T} \mathbf{E}^{T} \mathbf{f}_{i j}+\mathbf{c}_{l j}^{T} \mathbf{E}^{T} \mathbf{f}_{c l j}+\mathbf{c}_{r j}^{T} \mathbf{E}^{T} \mathbf{f}_{c r j},
$$

with

$$
\mathbf{E}=\left[\begin{array}{cc}
0 & -1 \\
1 & 0
\end{array}\right]
$$


where $\mathbf{a}_{i j}=\left[\begin{array}{ll}a_{i j}^{x} & a_{i j}^{y}\end{array}\right]^{T}$ denotes the Cartesian coordinate vectors of point $A_{i j}, \mathbf{c}_{l j}=\left[\begin{array}{ll}c_{l j}^{x} & c_{l j}^{y}\end{array}\right]^{T}$ and $\mathbf{c}_{r j}=\left[\begin{array}{ll}c_{r j}^{x} & c_{r j}^{y}\end{array}\right]^{T}$ denote the Cartesian coordinate vectors of contact points $C_{l j}$ and $C_{r j}$, respectively. $\mathbf{g}_{j}=\left[\begin{array}{ll}g_{j}^{x} & g_{j}^{y}\end{array}\right]^{T}$ is the Cartesian coordinate vector of the center of gravity $G_{j}$. Let $m_{C r j}$ be the moment generated at the right contact point $C_{r j}$ at the instant when $\mathscr{M}_{j}$ loses contact with the ground at point $C_{l j}$ such that $f_{c_{l j}}^{y}=0$, expressed as:

$$
m_{C r j}=\left(\mathbf{g}_{j}-\mathbf{c}_{r j}\right)^{T} \mathbf{E}^{T} \mathbf{w}_{g j}+\sum_{i=1}^{q_{j}}\left(\mathbf{c}_{r j}-\mathbf{a}_{i j}\right)^{T} \mathbf{E}^{T} \mathbf{t}_{i j}
$$

Let $\Sigma_{j}$ be the set composed of $\mathscr{M}_{j}$, its front and rear wheels, the cables attached to it and the point-mass end-effector, as encircled in red in Fig. 2. From the free body diagram of $\Sigma_{i}$, moment $m_{C r j}$ can also expressed as:

$$
\begin{aligned}
m_{C r j}= & -\left(\mathbf{p}-\mathbf{c}_{r j}\right)^{T} \mathbf{E}^{T} \mathbf{f}+\left(\mathbf{g}_{j}-\mathbf{c}_{r j}\right)^{T} \mathbf{E}^{T} \mathbf{w}_{g j} \\
& +\sum_{o=1, o \neq j}^{p} \sum_{i=1}^{q_{o}}\left(\mathbf{p}-\mathbf{c}_{r j}\right)^{T} \mathbf{E}^{T} \mathbf{t}_{i o},
\end{aligned}
$$

where $o=1, \ldots, p$ and $o \neq j$. p denotes the Cartesian coordinate vector of the point-mass end-effector $P . \mathbf{f}=\left[\begin{array}{ll}f^{x} & f^{y}\end{array}\right]^{T}$ denotes the force applied by the cables onto the point-mass end-effector, namely,

$$
\mathbf{f}=-\mathbf{f}_{e} .
$$

Similarly, the moment $m_{C l j}$ generated at the left contact point $C_{l j}$ on $\Sigma_{j}$ takes the form:

$$
\begin{aligned}
m_{C l j}= & -\left(\mathbf{p}-\mathbf{c}_{l j}\right)^{T} \mathbf{E}^{T} \mathbf{f}+\left(\mathbf{g}_{j}-\mathbf{c}_{l j}\right)^{T} \mathbf{E}^{T} \mathbf{w}_{g j} \\
& +\sum_{o=1, o \neq j}^{p} \sum_{i=1}^{q_{o}}\left(\mathbf{p}-\mathbf{c}_{l j}\right)^{T} \mathbf{E}^{T} \mathbf{t}_{i o} .
\end{aligned}
$$

For $\mathscr{M}_{j}$ to be stable, the moments generated by the external forces at point $C_{r j}\left(C_{l j}\right.$, resp.) should be counterclockwise (clockwise, resp.), namely,

$$
\begin{aligned}
& m_{C r j} \geq 0, \quad j=1, \ldots, p \\
& m_{C l j} \leq 0, \quad j=1, \ldots, p
\end{aligned}
$$

\section{Available Wrench Set for MCDPRs}

In this section the nature of the AWS for MCDPRs is analyzed. The cable tension $t_{i j}$ associated with the $i$ th cable mounted on $\mathscr{M}_{j}$ is bounded between a minimum tension $t_{i j}$ and a maximum tension $\bar{t}_{i j}$. It should be noted that the AWS of a classical CDPR depends uniquely on its platform pose and cable tension limits and forms a zonotope [13]. In contrast, the tipping constraints of the MBs must be considered in the definition of the AWS of a MCDPR. The AWS $\mathscr{A}_{1}$ for a planar CDPR with a point mass end-effector can be expressed as:

$$
\begin{aligned}
\mathscr{A}_{1}= & \left\{\mathbf{f} \in \mathbb{R}^{2} \mid \mathbf{f}=\sum_{j=1}^{p} \sum_{i=1}^{q_{j}} t_{i j} \mathbf{u}_{i j}, \underline{t}_{i j} \leq t_{i j} \leq \bar{t}_{i j},\right. \\
& \left.i=1, \ldots, q_{j}, j=1, \ldots, p\right\} .
\end{aligned}
$$
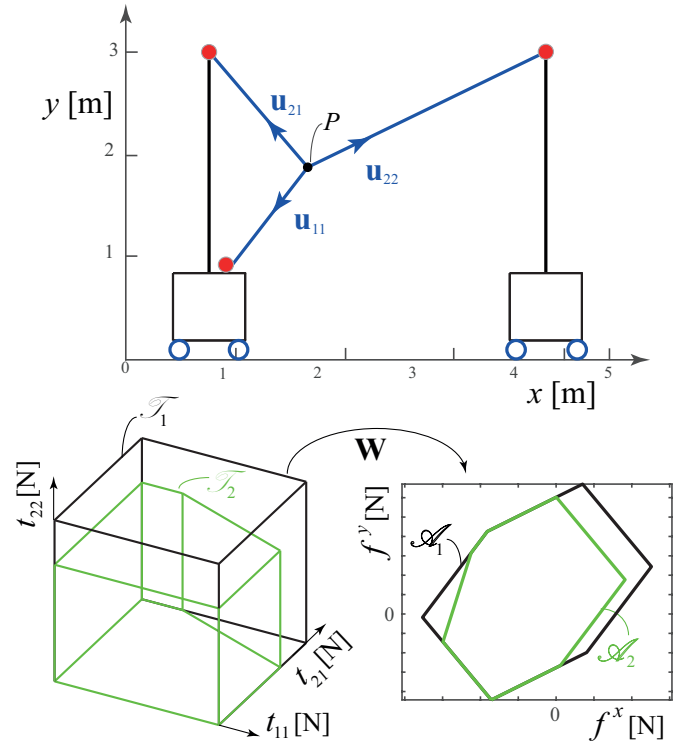

Fig. 3: MCDPR with $q=3$ cables and a $n=2$ DoF pointmass end-effector, the black polytopes illustrate the TS and AWS of the CDPR at hand, whereas green polytopes illustrate the TS and AWS of the MCDPR at hand

The AWS $\mathscr{A}_{2}$ for a planar MCDPR with a point-mass endeffector is derived from $\mathscr{A}_{1}$ by adding the tipping constraints defined in (19) and (20). Thus $\mathscr{A}_{2}$ can be expressed as:

$$
\begin{aligned}
\mathscr{A}_{2}= & \left\{\mathbf{f} \in \mathbb{R}^{2} \mid \mathbf{f}=\sum_{j=1}^{p} \sum_{i=1}^{q_{j}} t_{i j} \mathbf{u}_{i j}, \underline{t}_{i j} \leq t_{i j} \leq \bar{t}_{i j},\right. \\
& \left.m_{C r j} \geq 0, m_{C l j} \leq 0, i=1, \ldots, q_{j}, j=1, \ldots, p\right\} .
\end{aligned}
$$

Figure 3 shows a comparison between the Tension space (TS) $\mathscr{T}_{1}$ and the AWS $\mathscr{A}_{1}$ of a CDPR with three cables and an point-mass platform with the TS $\mathscr{T}_{2}$ and AWS $\mathscr{A}_{2}$ of a MCDPR with three cables, a point-mass platform and two mobile bases. The tension space between $t_{11}$ and $t_{21}$ is reduced by the linear tipping constraint on $\mathscr{M}_{1}$. As $\mathscr{M}_{2}$ is carrying a single cable $\mathscr{C}_{22}$, only the maximum limit $\bar{t}_{22}$ is modified. The difference in the polytopes $\mathscr{A}_{1}$ and $\mathscr{A}_{2}$ is due to the additional constraints associated with the equilibrium of the MCDPR MBs expressed by (19) and (20).

By considering only the classical cable tension limit $\left(t_{i j}\right.$ and $\left.\bar{t}_{i j}\right)$ constraints, the shape of the AWS is a zonotope. When the tipping constraints are included, the AWS is no longer a zonotope, but a convex polytope. The two methods used to represent convex polytopes are V-representation, known as the convex hull approach, and H-representation, known as the hyperplane shifting method [10]. V-representation is preferred for visualization while $\mathrm{H}$-representation is used to find the relation between $\mathscr{A}$ and $\mathscr{R}$. The convex-hull approach is used to find the vertices that form the boundary of the polytope, whereas hyperplane shifting method is a geometric method used to obtain the facets of the polytope. 

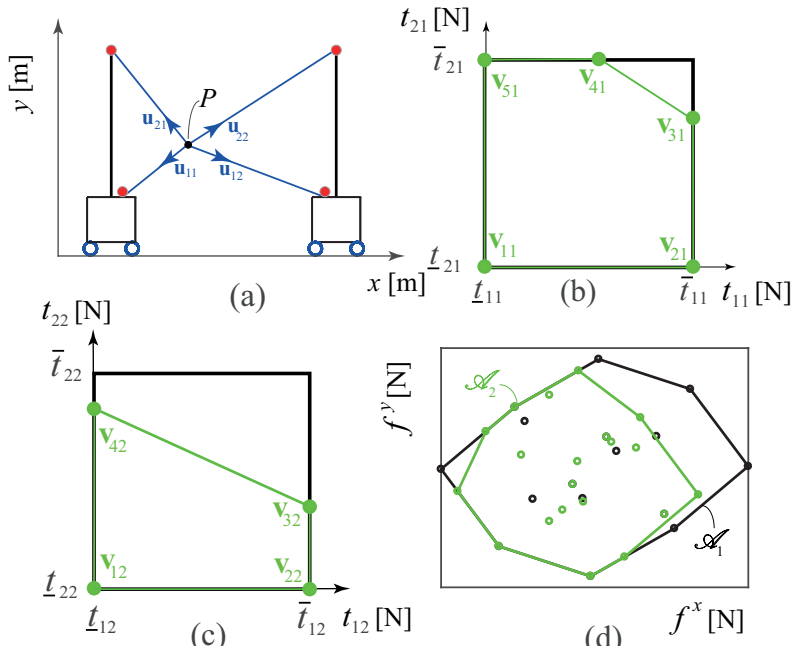

(c)

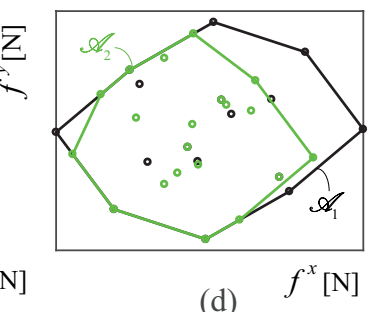

(d)

Fig. 4: Comparison of TS and AWS between CDPR (in black) and MCDPR (in green) (a) MCDPR configuration with $q_{1}=q_{2}=2$ (b) TS formed by $t_{i 1}$ (c) TS formed by $t_{i 2}$ (d) $\mathscr{A}_{1}$ is AWS of the CDPR at hand, $\mathscr{A}_{2}$ is AWS of the MCDPR at hand

\section{A. Convex Hull Method}

AWS is defined using the set of vertices forming the extreme points of the polytope. For the $j$ th mobile base $\mathscr{M}_{j}$, a $q_{j}$ dimensional TS is formed by the attached cables. The shape of this TS depends on the mapping of the tipping constraints on the $q_{j}$-dimensional TS formed by the cable tension limits $\underline{t}_{i j}$ and $\bar{t}_{i j}$. Figures 4(b) and 4(c) illustrate the TS associated with each MB of the MCDPR configuration shown in Fig. 4(a). The feasible TS is formed by the cable tension limits as well as the tipping constraints of the MBs. The new vertices of the TS for MCDPRs do not correspond with the minimum/maximum cable tensions as in the classical case [13].

Let $v_{j}$ denote the number of vertices and $\mathbf{v}_{k j}$ be the coordinates of $k$ th vertex for the TS associated to the $j$ th mobile base $\mathscr{M}_{j}, k=1, \ldots, v_{j}$. Let $\mathscr{V}_{j}$ represent the set of the vertices of the TS associated to $\mathscr{M}_{j}$ :

$$
\mathscr{V}_{j}=\left\{\mathbf{v}_{k j}\right\}, \quad k=\left\{1, \ldots, v_{j}\right\} .
$$

Let $\mathbf{V}_{j}$ be a $\left(q_{j} \times v_{j}\right)$ matrix containing the coordinates of the TS vertices associated with $\mathscr{M}_{j}$, expressed as:

$$
\mathbf{V}_{j}=\left[\begin{array}{llll}
\mathbf{v}_{1 j} & \mathbf{v}_{2 j} & \ldots & \mathbf{v}_{v_{j} j}
\end{array}\right] .
$$

$v$ is the total number of vertices formed by all the $q$ cables and is obtained by the product of the number of vertices for each MB, namely,

$$
v=\prod_{j=1}^{p} v_{j} .
$$

Let $\mathscr{V}$ represent the set of all vertices in the TS which is obtained by the Cartesian product between $\mathscr{V}_{j}, j=1, \ldots, p$. Accordingly, $\mathbf{V}$ is $(q \times v)$-matrix, which denotes the coordinates of all the vertices in $\mathscr{V}$ expressed as:

$$
\mathbf{V}=\left[\begin{array}{llllll}
\underline{\mathbf{v}}_{1} & \underline{\mathbf{v}}_{2} & \cdots & \underline{\mathbf{v}}_{g} & \cdots & \underline{\mathbf{v}}_{v}
\end{array}\right]
$$

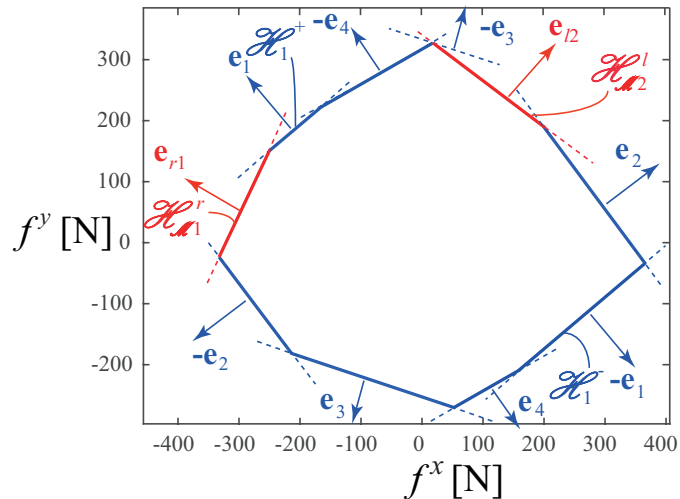

Fig. 5: AWS formed by the intersection of all the hyperplanes (red hyperplanes corresponds to the tipping constraints, blue hyperplanes corresponds to the cables tension limits)

where $g=1, \ldots, v . \underline{\mathbf{v}}_{g}$ is a $q$-dimensional vector representing the coordinates of the $g$ th vertex of the MCDPR Tension Space. The image of AWS is constructed from $\mathbf{V}$ under the mapping of the wrench matrix W. A numerical procedure such as quickhull [15] is used to compute the convex hull forming the boundary of AWS. Figure 4(d) illustrates the AWS obtained by Convex Hull Method. $\mathscr{A}_{1}$ is the AWS obtained by considering only the cable tension limits and is a zonotope. $\mathscr{A}_{2}$ is the AWS obtained by considering both the cable tension limits and the tipping constraints of the two mobile bases.

\section{B. Hyperplane Shifting Method}

Hyperplane Shifting Method (HFM) is a geometric approach, which defines a convex polytope as the intersection of the half-spaces bounded by its hyperplanes [14]. The classical HFM used to obtain the AWS of CDPRs is described in [13], [14]. Nevertheless, this approach is not sufficient to fully characterize the AWS of MCDPRs because it requires a hypercube TS $\left(\mathscr{T}_{1}\right)$. For instance, for the MCDPR shown in Fig. 3, it can be observed that the TS $\left(\mathscr{T}_{2}\right)$ is no longer a hypercube due to the additional constraints associated with the SE of the mobile bases.

As a consequence, this section presents an improved version of the HFM described in $[1,2]$ that takes into account the tipping constraints of the MCDPR mobile bases. As a result, Fig. 5 depicts the AWS of the MCDPR configuration shown in Fig. 4(a), obtained by the improved HFM. This AWS is bounded by hyperplanes $\mathscr{H}_{s}^{+}, \mathscr{H}_{s}^{-}, s=1, \ldots, q$, obtained from the cable tension limits associated to the four cables attached to the point mass end-effector, and by hyperplanes $\mathscr{H}_{\mathscr{M} 1}^{r}$ and $\mathscr{H}_{\mathscr{M} 2}^{l}$, corresponding to the tipping constraints of $\mathscr{M}_{1}$ about point $C_{r 1}$ and the tipping constraint of $\mathscr{M}_{2}$ about point $C_{l 2}$, respectively.

1) Determination of $\mathscr{H}_{\mathscr{M} j}^{l}$ and $\mathscr{H}_{\mathscr{M} j}^{r}, j=1, \ldots, p$ : Let $\mathbf{r}_{r j}$ $\left(\mathbf{r}_{l j}\right.$, resp.) be the unit vector pointing from $C_{r j}\left(C_{l j}\right.$, resp.) to $P$, expressed as:

$$
\mathbf{r}_{r j}=\frac{\mathbf{p}-\mathbf{c}_{r j}}{\left\|\mathbf{p}-\mathbf{c}_{r j}\right\|_{2}}, \quad \mathbf{r}_{l j}=\frac{\mathbf{p}-\mathbf{c}_{l j}}{\left\|\mathbf{p}-\mathbf{c}_{l j}\right\|_{2}} .
$$

Upon dividing (19) ((20), resp.) by $\left\|\mathbf{p}-\mathbf{c}_{r j}\right\|_{2}\left(\left\|\mathbf{p}-\mathbf{c}_{l j}\right\|_{2}\right.$, 
resp.), the tipping constraints can be expressed in the wrench space as:

$$
\begin{aligned}
& -\mathbf{r}_{r j}^{T} \mathbf{E}^{T} \mathbf{f}+\frac{\left(\mathbf{g}_{j}-\mathbf{c}_{r j}\right)^{T}}{\left\|\mathbf{p}-\mathbf{c}_{r j}\right\|_{2}} \mathbf{E}^{T} \mathbf{w}_{g j}+\sum_{o=1, o \neq j}^{p} \sum_{i=1}^{q_{o}} \mathbf{r}_{r j}^{T} \mathbf{E}^{T} \mathbf{t}_{i o} \geq 0, \\
& -\mathbf{r}_{l j}^{T} \mathbf{E}^{T} \mathbf{f}+\frac{\left(\mathbf{g}_{j}-\mathbf{c}_{l j}\right)^{T}}{\left\|\mathbf{p}-\mathbf{c}_{l j}\right\|_{2}} \mathbf{E}^{T} \mathbf{w}_{g j}+\sum_{o=1, o \neq j}^{p} \sum_{i=1}^{q_{o}} \mathbf{r}_{l j}^{T} \mathbf{E}^{T} \mathbf{t}_{i o} \leq 0 .
\end{aligned}
$$

Equations (28) and (29) take the form:

$$
\mathbf{e}_{r j}^{T} \mathbf{f} \leq d_{r j}, \quad \mathbf{e}_{l j}^{T} \mathbf{f} \leq d_{l j}
$$

Equation (30) corresponds to the hyperplanes [14] for the tipping constraints of $\mathscr{M}_{j}$ in the wrench space. $\mathbf{e}_{r j}$ and $\mathbf{e}_{l j}$ are the unit vectors normal to $\mathscr{H}_{\mathscr{M} j}^{r}$ and $\mathscr{H}_{\mathscr{M} j}^{l}$, expressed as:

$$
\mathbf{e}_{r j}=\mathbf{E r}_{r j}, \quad \mathbf{e}_{l j}=-\mathbf{E r}_{l j}
$$

$d_{r j}\left(d_{l j}\right.$, resp.) denotes the shifted distance of $\mathscr{H}_{j}^{r}\left(\mathscr{H}_{j}^{l}\right.$, resp.) from the origin of the wrench space along $\mathbf{e}_{r j}\left(\mathbf{e}_{l j}\right.$, resp.). The shift $d_{r j}$ depends on the weight of $\mathscr{M}_{j}$ and the combination of the cable tension $t_{i o}$ for which $\sum_{o=1, o \neq j}^{p} \sum_{i=1}^{q_{o}} \mathbf{r}_{r j}^{T} \mathbf{E}^{T} \mathbf{t}_{i o}$ is a maximum. While the shift $d_{r l}$ depends on the weight of $\mathscr{M}_{j}$ and the combination of the cable tension $t_{i o}$ for which $\sum_{o=1, o \neq j}^{p} \sum_{i=1}^{q_{o}} \mathbf{r}_{l j}^{T} \mathbf{E}^{T} \mathbf{t}_{i o}$ is a minimum, namely,

$$
\begin{aligned}
d_{r j}= & \frac{\left(\mathbf{g}_{j}-\mathbf{c}_{r j}\right)^{T}}{\left\|\mathbf{p}-\mathbf{c}_{r j}\right\|_{2}} \mathbf{E}^{T} \mathbf{w}_{g j} \\
& +\sum_{o=1, o \neq j}^{p} \max \left(\sum_{i=1}^{q_{o}} \mathbf{r}_{r j}^{T} \mathbf{E}^{T} v_{k o}^{i} \mathbf{u}_{i o}, k=1, \ldots, v_{o}\right), \\
d_{l j}= & -\frac{\left(\mathbf{g}_{j}-\mathbf{c}_{l j}\right)^{T}}{\left\|\mathbf{p}-\mathbf{c}_{l j}\right\|_{2}} \mathbf{E}^{T} \mathbf{w}_{g j} \\
& -\sum_{o=1, o \neq j}^{p} \min \left(\sum_{i=1}^{q_{o}} \mathbf{r}_{l j}^{T} \mathbf{E}^{T} v_{k o}^{i} \mathbf{u}_{i o}, k=1, \ldots, v_{o}\right),
\end{aligned}
$$

where $v_{k o}^{i}$ corresponds to the $i$ th coordinate of $\mathbf{v}_{k o}$. Figure 6 shows the geometric representation of the tipping hyperplanes for the MCDPR under study. From Figs. 5 and 6, it can be observed that the orientation of $\mathscr{H}_{\mathscr{M} j}^{r}\left(\mathscr{H}_{\mathscr{M} j}^{l}\right.$, resp.) is directly obtained from $\mathbf{r}_{r j}\left(\mathbf{r}_{l j}\right.$, resp.)

2) Determination of $\mathscr{H}_{s}^{+}$and $\mathscr{H}_{s}^{-}, s=1, \ldots, q$ : For classical CDPRs with given cable tension limits, $\Delta t_{i j}=\bar{t}_{i j}-\underline{t}_{i j}$ is a constant, AWS is a zonotope formed by the set of vectors $\alpha_{i j} \Delta t_{i j} \mathbf{u}_{i j}$, where $0 \leq \alpha_{i j} \leq 1$ [13], [14]. The shape of the zonotope depends on the directions of the cable unit vectors $\mathbf{u}_{i j}$ as well as the difference between the minimum and maximum cable tension limits $\Delta t_{i j}$. It is noteworthy that $\Delta t_{i j}$ is no longer a constant for MCDPRs. The property of a zonotope having parallel facets still holds as the orientation of the hyperplanes is given by the cable unit vectors $\mathbf{u}_{i j}$. However, the position of the hyperplanes is modified, forming a convex polytope with parallel facets rather than a zonotope.

$\mathscr{H}_{s}^{+}$and $\mathscr{H}_{s}^{-}$are obtained using the classical HFM as described in [13], [14] based on the TS of MCDPRs. For a

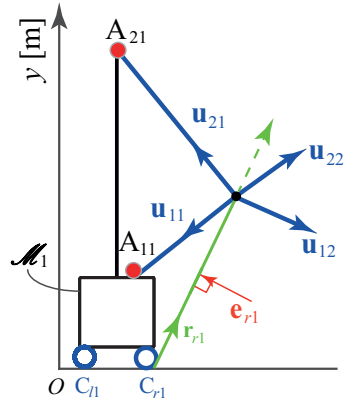

(a)

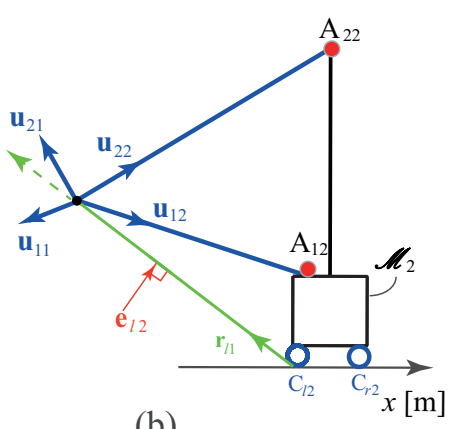

(b)
Fig. 6: Geometric representation of $\mathbf{e}_{r 1}$ and $\mathbf{e}_{l 2}$ from the MCDPR configuration

planar MCDPR with a point mass end-effector, each cable unit vector $\mathbf{u}_{s}=\mathbf{u}_{i j}$ will form a pair of parallel hyperplanes $\left\{\mathscr{H}_{s}^{+}, \mathscr{H}_{s}^{-}\right\}$at the origin. Each pair is associated with a unit vector $\mathbf{e}_{s}$ orthogonal to its facets, expressed as:

$$
\mathbf{e}_{s}=\mathbf{E}^{T} \mathbf{u}_{s} .
$$

The shift of the initial hyperplanes is determined by the projection of the MCDPR tension space vertices on $\mathbf{e}_{s}$. Let $\mathbf{l}_{s}$ be a $q$-dimensional vector containing the projections of the cable unit vectors in $\mathbf{W}$ on $\mathbf{e}_{s}$, expressed as:

$$
\mathbf{l}_{s}=\mathbf{W}^{T} \mathbf{e}_{s} .
$$

The projection of $\mathbf{u}_{s}$ will be zero as it is orthogonal to $\mathbf{e}_{s}$. The distances $h_{s}^{+}$and $h_{s}^{-}$are given by the maximum and minimum combinations of $\mathbf{l}_{s}$ with the coordinates of the TS vertices $\mathbf{V}$, expressed as:

$$
\begin{aligned}
& h_{s}^{+}=\max \left(\sum_{s=1}^{q} \underline{v}_{g}^{s} l^{s}, \quad g=1, \ldots, v\right), \\
& h_{s}^{-}=\min \left(\sum_{s=1}^{q} \underline{v}_{g}^{s} l^{s}, \quad g=1, \ldots, v\right),
\end{aligned}
$$

where $\underline{v}_{g}^{s}$ and $l^{s}$ denote the sth coordinate of vector $\underline{\mathbf{v}}_{g}$ and $\mathbf{l}_{s}$, respectively. To completely characterize the hyperplanes, a

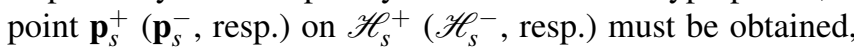
given as:

$$
\mathbf{p}_{s}^{+}=h_{s}^{+} \mathbf{e}_{s}+\sum_{j=1}^{p} \sum_{i=1}^{q_{j}} \underline{t}_{i j} \mathbf{u}_{i j} ; \quad \mathbf{p}_{s}^{-}=h_{s}^{-} \mathbf{e}_{s}+\sum_{j=1}^{p} \sum_{i=1}^{q_{j}} \underline{t}_{i j} \mathbf{u}_{i j},
$$

where $\sum_{j=1}^{p} \sum_{i=1}^{q_{j}} t_{i j} \mathbf{u}_{i j}$ is the wrench generated by the minimum acceptable tension in all the cables. The corresponding shifts $d_{s}^{+}$and $d_{s}^{-}$of $\mathscr{H}_{s}^{+}$and $\mathscr{H}_{s}^{-}$are calculated as:

$$
d_{s}^{+}=\mathbf{e}_{s}^{T} \mathbf{p}_{s}^{+} ; \quad d_{s}^{-}=\mathbf{e}_{s}^{T} \mathbf{p}_{s}^{-} .
$$

The respective pair of hyperplanes is expressed as:

$$
\mathscr{H}_{s}^{+}: \mathbf{e}_{s}^{T} \mathbf{f} \leq d_{s}^{+} ; \quad \mathscr{H}_{s}^{-}: \quad-\mathbf{e}_{s}^{T} \mathbf{f} \leq d_{s}^{-} .
$$

The above procedure is repeated to determine the $q$ pairs of hyperplanes associated to the $q$ cables of the MCDPR. 


\section{WORKSPACE ANALYSIS}

Wrench-feasible workspace (WFW) is defined as the set of poses that are wrench-feasible [16]. A well known index used to compute the wrench feasible set of poses is called Capacity Margin [11], [12]. It is a measure of the robustness of the equilibrium of the robot, expressed as:

$$
s=\min \left(\min s_{j, l}\right),
$$

where $s_{j, l}$ is the signed distance from $j$ th vertex of the required wrench set $\mathscr{R}$ to the $l$ th face of the available wrench set $\mathscr{A} . s_{j, l}$ is positive when the constraint is satisfied, and negative otherwise. The index remains negative as long as at least one of the vertices of $\mathscr{R}$ is outside of $\mathscr{A}$. The index is positive if all the the vertices of $\mathscr{R}$ are inscribed by $\mathscr{A}$.

\section{EXPERIMENTS AND RESULTS}

The proposed approach was tested on MCDPR prototype shown in Fig. 7(a), made up of two TurtleBot mobile bases and a $0.5 \mathrm{~kg}$ point mass end-effector. The WFW of the MCDPR under study is illustrated in Fig. 7(b) for $\mathscr{R}$ equal to the weight of the end-effector. The green region corresponds to the modified WFW where both cable tension limits and mobile base tipping constraints are satisfied. In blue area, at least one of the two mobile bases is tipping. In red area, both the cable tension limits and the mobile base tipping constraints are not satisfied to keep the end-effector in equilibrium and to avoid mobile base tipping. It can be observed that for MCDPRs, the ability of the cables to apply wrenches on the platform may be reduced due to the mobile base tipping constraints. The simulation and the experimental validation of the proposed approach can be seen in video ${ }^{1}$. In the latter, two different trajectories are tested and compared. Based on the proposed workspace analysis, it can be observed that if the end-effector is within the defined WFW calculated offline, both the mobile bases are in equilibrium. On the contrary, for the end-effector outside of the WFW, at least one mobile base is not in equilibrium anymore.

\section{CONCLUSION}

In this paper, the Available Wrench Set required to trace the Wrench Feasible Workspace of a Mobile Cable-Driven Parallel Robot (MCDPR) has been determined. The proposed workspace considers the cable tension limits and the static equilibrium of the mobile bases. Two different approaches, the convex hull and the hyperplane shifting method, are used to illustrate how the additional constraints can be considered. The additional constraints modified the shape of the AWS, forming new facets and reducing the capability of the cables to apply wrenches on the platform. Future work will focus on extending this approach to spatial MCDPRs consisting of more than two mobile bases and taking into account wheel slipping constraints. Furthermore, the evolution of the MCDPR workspace during their deployment will be studied.

\footnotetext{
${ }^{1}$ https://youtu.be/9fvJY2nyLZY
}

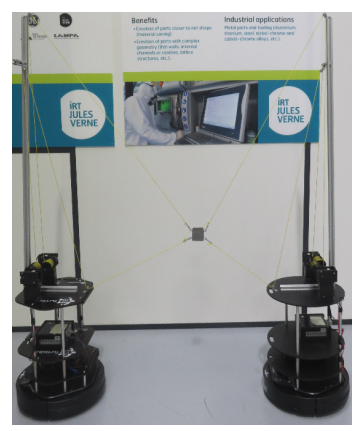

(a)

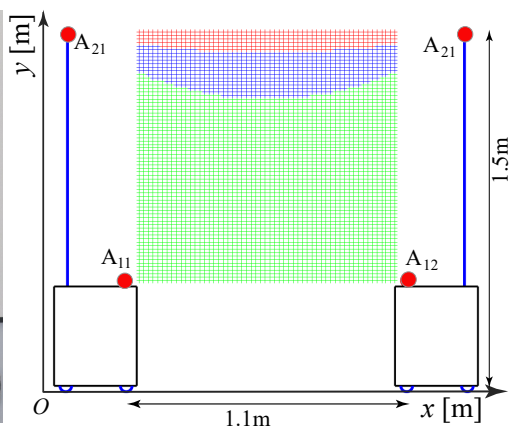

(b)
Fig. 7: MCDPR prototype made up of two TurtleBot mobile bases and a $0.5 \mathrm{~kg}$ point mass end-effector

\section{REFERENCES}

[1] Rasheed, T., Long, P., Marquez-Gamez, D. and Caro, S., Tension Distribution Algorithm for Planar Mobile Cable-Driven Parallel Robots, The Third International Conference on Cable-Driven Parallel Robots (CableCon 2017), Quebec City, Canada, August 242017.

[2] Pott, A., Mütherich, H., Kraus, W., Schmidt, V., Miermeister, P., Verl, A.: IPAnema: a family of cable-driven parallel robots for industrial applications. In: Cable-Driven Parallel Robots, Mechanisms and Machine Science, vol. 12, pp. 119134. Springer (2013)

[3] L. Gagliardini, S. Caro, M. Gouttefarde, and A. Girin. A reconfiguration strategy for reconfigurable cable-driven parallel robots. In 2015 IEEE International Conference on Robotics and Automation (ICRA), pages 1613-1620. IEEE, 2015.

[4] Gagliardini, L., Caro, S., Gouttefarde, M. and Girin, A., 2016. Discrete reconfiguration planning for cable-driven parallel robots. Mechanism and Machine Theory, 100, pp.313-337.

[5] Nguyen, D.Q., Gouttefarde, M., Company, O. and Pierrot, F., 2014, May. On the analysis of large-dimension reconfigurable suspended cable-driven parallel robots. In 2014 IEEE International Conference on Robotics and Automation (ICRA), pages 5728-5735. IEEE 2014.

[6] Rosati, G., Zanotto, D. and Agrawal, S.K., 2011. On the design of adaptive cable-driven systems. Journal of mechanisms and robotics, 3(2), p.021004.

[7] Gouttefarde, M. and Gosselin, C.M., 2006. Analysis of the wrenchclosure workspace of planar parallel cable-driven mechanisms. IEEE Transactions on Robotics, 22(3), pp.434-445.

[8] D. Lau, D. Oetomo, and S. K. Halgamuge, Wrench-Closure Workspace Generation for Cable Driven Parallel Manipulators using a Hybric Analytical-Numerical Approach, ASME J. Mech. Des., Vol. 133, 071004, 2011.

[9] Gouttefarde, M., Daney, D. and Merlet, J.P., 2011. Interval-analysisbased determination of the wrench-feasible workspace of parallel cable-driven robots. IEEE Transactions on Robotics, 27(1), pp.1-13.

[10] Grünbaum, B., 2003, Convex Polytopes, Springer, New York

[11] Guay, F., Cardou, P., Cruz-Ruiz, A.L. and Caro, S., 2014. Measuring how well a structure supports varying external wrenches. In New Advances in Mechanisms, Transmissions and Applications (pp. 385392). Springer, Dordrecht.

[12] Ruiz, A.L.C., Caro, S., Cardou, P. and Guay, F., 2015. Arachnis: Analysis of robots actuated by cables with handy and neat interface software. In Cable-Driven Parallel Robots (pp. 293-305). Springer International Publishing.

[13] Bouchard, S., Gosselin, C. and Moore, B., 2010. On the ability of a cable-driven robot to generate a prescribed set of wrenches. Journal of Mechanisms and Robotics, 2(1), p.011010.

[14] Gouttefarde, M. and Krut, S., 2010. Characterization of parallel manipulator available wrench set facets. Advances in robot kinematics: motion in man and machine, pp.475-482.

[15] Barber, C.B., Dobkin, D.P. and Huhdanpaa, H., 1996. The quickhull algorithm for convex hulls. ACM Transactions on Mathematical Software (TOMS), 22(4), pp.469-483

[16] Bosscher, P., Riechel, A.T. and Ebert-Uphoff, I., 2006. Wrenchfeasible workspace generation for cable-driven robots. IEEE Transactions on Robotics, 22(5), pp.890-902. 